\title{
Urinary System Findings Completion
}

\section{Status}

National Cancer Institute

\section{Source}

National Cancer Institute. Urinary System Findings Completion Status. NCI Thesaurus.

Code C162338.

A term used to describe the state or condition of the completeness of the urinary system findings data. 